\title{
Recent Changes in the Infant Mortality Rates of Blacks and Whites in the United States
}

\section{Rati Ram*}

Economics Department, Illinois State University, IL 61790-4200, USA

*Corresponding author: Rati Ram, Economics Department, Illinois State University, IL 61790-4200, USA, Tel: 3094387101; E-mail: rram@ilstu.edu

Received date: May 08, 2017; Accepted date: May 13, 2017; Published date: May 20, 2017

Copyright: (c) 2017 Ram R. This is an open-access article distributed under the terms of the Creative Commons Attribution License, which permits unrestricted use, distribution, and reproduction in any medium, provided the original author and source are credited.

Abstract
In the context of large differences in the Infant Mortality Rates (IMRs) of Blacks and Whites in the United States,
changes in the rates for the two groups over the period $2007-2014$ are compared. The comparisons indicate four
main points. First, the declines for the entire United States are fairly similar at $16.7 \%$ and $14.3 \%$ for Blacks and
Whites respectively. Second, the excess of decline in Black IMR during the period is considerably smaller than that
during $2000-2007$ when the declines were $6.4 \%$ and $1.8 \%$ for Blacks and Whites respectively. Third, there is huge
diversity in the levels of IMR, and the rates of declines, across the U.S. states and the two races. For example, of
the 34 states for which comparisons could be made, the decline in Black IMR is smaller than in White IMR for 13
states, including Alabama, Arizona, California, Connecticut, Florida, Georgia, Minnesota, New York, Ohio,
Pennsylvania and Washington. There are two states (Alabama and Washington) where Black IMR increased during
the period while White IMR declined. Fourth, therefore, the US government's goal of eliminating racial disparities in
IMRs (and other health indicators) is unlikely to be reached in the foreseeable future. Consequently, the position of
the United States in the global rankings of IMRs is unlikely to improve much in the near future.

Keywords: Infant Mortality Rates (IMRs); Black and White comparisons

\section{Introduction}

The infant mortality rate (IMR) in the United States is known to have been high relative to other developed countries and even relative to many developing countries. For example, estimates for 2016 by the US Central Intelligence Agency [1] show that IMR for the United States at 5.80 (per thousand live births) ranks 169 among 225 countries or territories. The Unites States is thus behind 56 countries which include not merely most developed countries, but also others like Latvia (5.30), Slovakia (5.20), Hungary (5.00), Estonia (3.80), Bermuda (2.50) and Monaco (1.80).

One reason for the high IMR in the United States is the very large disparity in the IMRs for Blacks and Whites. For example, while the non-Hispanic White IMR for 2014 at 4.8 [2] might not look too bad, the Black IMR is 11.0 , and at 2.29 times the White IMR, pulls the United States down in the international ranking.

An enormous amount of research has been devoted to the study of Black- White IMR differential, including its causes, consequences, and possible remedial measures. However, "despite hundreds of studies addressing this issue, researchers have been unable to explain why African American infants are at greater risk for low birth weights, intrauterine growth restriction, (and) prematurity" [3]. Reflecting the public health relevance of this phenomenon, Healthy People 2010 [4] called for an elimination of the racial disparities in infant mortality rates. Ram [5] also explored this phenomenon at considerable length and indicated that, considering the past trends, the goal of eliminating Black-White IMR disparity was unlikely to be met for a long time. He also suggested that (a) the observed pattern seemed to reflect poorer access of Blacks to prenatal, maternal and infant healthcare, and (b) the disparities may be partly due to a heavy reliance on the market forces in healthcare provision and innovation, particularly relative to infant mortality.

Since the last year covered by Ram's study [5] was 2007, it is of interest to consider the pattern after that year. This research is an effort in that direction. It compares Black and White IMRs for 2007 with those for 2014 which is the most recent year for which the data are available. The comparison is done for the United States and also for each state for which relevant data are available. The comparisons are done primarily in terms of the rates of change in the IMRs from 2007 to 2014. For the United States, a quick comparison is also done of the change from 2007 to 2014 with that from 2000 to 2007 . The general pattern indicated by the comparisons is that there is little indication of a significant reduction in the Black-White IMR disparity. On the contrary, the rate of reduction in the gap during the period 2000-2007 was larger than that during 2007-2014, and there seems a slowdown in the rate of reduction in the Black-White IMR gap. Several related aspects are also briefly discussed.

\section{Methodology, data, and the main results}

A simple methodology is adopted. For the United States and each state, percent rate of change in the Black and the White IMR from 2007 to 2014 (CHANGE) is calculated from the following expression:

\section{CHANGE=[(IMR2014/IMR2007)-1 $] \times 100 \quad$ (1)}

Where IMR2014 is the IMR for 2014 for each unit (U.S. and each state) and race and IMR2007 is the corresponding rate for 2007. Since the IMRs are expected to decline over the period, the percentage change is expected to be a negative number in most cases, and a positive sign would indicate the IMR increased from 2007 to 2014.

Most data are taken from KIDS COUNT data center [2] where the original source is stated to be Centers for Disease Control and Prevention, National Center for Health Statistics. As already noted, 
since the period covered by Ram [5] ended with 2007, the data used in the present research are mainly from 2007 to 2014 .

Table 1 reports the rates of change in IMR from 2007 to 2014 for the United States and for each state for which data for both years are available in the cited source. The table indicates the following main points.

1. For the United States, the rate of decline in the IMR for Blacks is $16.7 \%$ over the 7 -year period while the corresponding rate for the Whites is slightly lower at $14.3 \%$. One might thus say that the two groups experienced broadly similar rates of decline, and the excess of Black IMR over the White IMR has not changed much. The ratio of Black and White IMRs in 2014 is 2.29 which is only a tiny decline from 2.36 in 2007, and the evidence for a tendency toward convergence in the IMRs for the two races is negligible. Taking a somewhat longer perspective, it is useful to note that decline in the Black-White IMR disparity during 2007-2014 is smaller than that during the preceding seven years. Statistical Abstract of the United States [6] shows Black and White IMRs to be 14.1 and 5.7 respectively in 2000 and 13.2 and 5.6 in 2007, implying a larger decline of $6.4 \%$ in the Black IMR as compared to a decline of $1.8 \%$ in the White IMR. Over the 14 -year period one may thus sense a slight slowdown in the reduction of BlackWhite IMR disparity, perhaps partly due to the financial crisis of 2009.

2. Looking at the changes in the 34 states for which comparable data are available, the first impression is that of a tremendous diversity in the levels of IMRs and their rates of change both across states and the two race groups. For example, apart from the increases in Alabama and Washington, the decline in the Black IMR varies from a low of $0.8 \%$ for Kentucky to a high of $32.1 \%$ in Missouri. For the White IMR the decline varies from 0 in Arkansas and Massachusetts to $43.1 \%$ for Connecticut. The differences in the rates of decline across the two races in each state are as stark. For example, in Alabama, Black IMR increased by $1.4 \%$ while the White IMR declined by $26.8 \%$. In California, while the Black IMR declined by $14.5 \%$, White IMR declined by $24.5 \%$. Similarly, in Connecticut the Black IMR declined at a much lower rate (18.2\%) than White IMR which declined by $43.1 \%$.

3. Considering the overall scenario, it may be noted that White IMR declined at a higher rate than Black IMR in 13 states, including such large states as California, Connecticut, Florida, Georgia, Minnesota New York, Ohio, and Washington, and the excess of White IMR decline is large in several cases, including Alabama, California, Connecticut, Georgia, and Texas. In the other 21 states, Black IMR declined at a higher rate than White IMR, and the excess of decline in the Black IMR is substantial in some cases, notably Iowa, Louisiana, Maryland, Massachusetts, Missouri, New Jersey, and Oklahoma. These divergent patterns across states generate the overall small excess of decline in Black IMR over that in White IMR. In other words, the small overall excess of Black IMR decline is not due to a similar pattern of declines across the states, but reflects the aggregation of extremely divergent scenarios across the states.

\section{Some related thoughts}

The first thought one is likely to have is that of the tremendous variations indicated by Table 1 in the IMRs and their rates of decline across the states and the two racial groups, some of which are summarized in the preceding section. Articulation of these huge variations across states and the two racial groups in both levels of IMRs and their rates of decline is perhaps a significant contribution of this study. While an analysis of the observed divergences is beyond the scope of this short paper, it should deserve careful consideration in future research. A grasp of the observed variations should be helpful toward a better understanding of the large Black-White IMR disparities in the United States and each of the states.

Although the focus of this paper is largely descriptive, it is of some interest to reflect briefly on the causes of the large Black-White IMR disparity.

Medically, as SACIM Subcommittee on Eliminating Health Disparities [3] noted, the disparity is mainly due to the excess among Black infants of low-weight and preterm births and intrauterine growth restriction as compared to White infants. However, as the authors of that Report stated, despite hundreds of studies, researchers have not been able to explain those differences in the Black and White infants. Ram [5] gave some consideration to the likely reasons and noted three possibilities. First, Blacks seem to have poorer access to prenatal, maternal and infant healthcare due to poverty and the socioeconomic structures. In this context, he noted the lower health insurance coverage of Blacks. In a somewhat similar tone, Mayer and Sarin [7] have articulated several mechanisms that link economic inequality and infant mortality. Second, Ram [5] attributed some of the weaker access of Blacks to healthcare to the dominant role of market forces in the provision of healthcare in the United States, and suggested that greater role of the public sector might reduce the Black-White IMR disparity. Third, he noted the perceptive analysis by Cutler et al. [8] who developed a model of induced innovation to show that, as a consequence of targeting the most common conditions in the population, induced innovation leads to an increase in mortality disparity between minority and majority groups. Using information on infant deaths in the United States between 1983 and 1998, they found empirical support for their model. This aspect can perhaps be related to Ram's broader point that heavy reliance on market forces for provision of healthcare and the related induced innovations may be a significant factor in the large Black-White IMR disparities.

Irrespective of the reasons for the large Black-White IMR gap, the trends summarized in the preceding section make one pessimistic that the Black IMR can be greatly reduced and the Black-White disparity can be eliminated in the foreseeable future. Therefore, it seems unlikely that the international ranking of the United States in regard to IMR will improve much in the near future.

It is perhaps useful to note that, although Black-White disparity in maternal mortality does not seem to have received much attention, that disparity is larger than in the IMRs for the two races. For example, Statistical Abstract of the United States 2012 [6] shows that in the year 2007, while maternal mortality rate for Whites was 10.0 (per thousand live births), the rate for Blacks was 26.5 with the implied ratio of 2.65 which is higher than the ratio of $2.36(13.2 / 5.6)$ for the IMRs for that year [6]. It seems maternal mortality may be affected as much as infant mortality by poorer access of Blacks to healthcare, heavy reliance on market forces, and possibly the nature of induced innovations.

It is perhaps also of some interest to note that Black-White disparity in child death rates is smaller than that in infant mortality or maternal mortality. KIDS COUNT data center [2] indicates the death rate for children between ages 1 and 14 (per 100,000 children in the age group) for the year 2014 as 23 for Blacks and 15 for non-Hispanic Whites, which is a ratio of about $1: 1.5$. Black-White mortality rate ratio for children age 1-4 is 1.64 [9] which also is much lower than the ratios for IMR and maternal mortality. This is an interesting point and indicates 
Citation: Rati R (2017) Recent Changes in the Infant Mortality Rates of Blacks and Whites in the United States. Health Econ Outcome Res 3:

Page 3 of 4

that IMR and maternal mortality are more affected by racial disparities in access to healthcare than child mortality.

Although the foregoing narrative may seem pessimistic about the Black- White IMR gap being eliminated in the foreseeable future, one positive aspect is of interest. Ram [5] indicated that while the BlackWhite IMR disparity had been increasing and the ratio went up from 2.04 in 1980 to 2.48 in 2000 , it declined from 2.48 in 2000 to 2.35 in 2007. That thought might, however, be mitigated by the slower decline during 2007-2014.

\section{Concluding Observations}

This short paper is a study of the recent patterns in Black and White IMRs in the United States. Its main contribution rests on five points. First, the big Black-White IMR disparity shows only a small decline from 2007 to 2014 and the reduction is less than that occurred during the preceding seven years (2000-2007). Second, a tremendous diversity is observed in the levels of IMRs and their rates of change across the
U.S. states and the two race groups. An understanding of this diversity seems to be an important area for future research on the topic. Third, a quick look is taken at the causes of the Black-White IMR disparity. While in medical terms, the disparity appears to arise mainly from higher low-weight and preterm births among Black infants, the more fundamental reasons may lie in the weaker access of Blacks to healthcare, their socio-economic disadvantage, and social and income inequality. In addition, heavy reliance on the market for healthcare provision and induced innovations may also be contributory factors. Fourth, it is noted that while Black-White maternal-mortality disparity is even larger than IMR disparity, the Black-White disparity in mortality of children is considerably smaller, indicating some resilience among children to the economic and social disadvantages that the Blacks might be subject to. Last, the extremely slow convergence in Black-White IMRs implies that the goal of eliminating racial health disparities is unlikely to be met in the foreseeable future, and the continuing high Black IMR would prevent a significant improvement in the international ranking of the United States in the near future.

\begin{tabular}{|c|c|c|c|c|c|c|}
\hline & \multicolumn{3}{|c|}{ Black IMR (per thousand births) } & \multicolumn{3}{|c|}{ White IMR (per thousand births } \\
\hline & 2007 & 2014 & Change (\%) & 2007 & 2014 & Change (\%) \\
\hline United States & 13.2 & 11 & -16.7 & 5.6 & 4.8 & -14.3 \\
\hline Alabama* & 14.4 & 14.6 & 1.4 & 8.2 & 6 & -26.8 \\
\hline Arizona* & 15 & 12.7 & -15.3 & 5.7 & 4.8 & -15.8 \\
\hline Arkansas & 13.2 & 11.9 & -9.8 & 6.7 & 6.7 & 0 \\
\hline California* & 12.4 & 10.6 & -14.5 & 4.9 & 3.7 & -24.5 \\
\hline Colorado & 13.2 & 11.2 & -15.2 & 4.9 & 4.4 & -10.2 \\
\hline Connecticut* $^{*}$ & 12.1 & 9.9 & -18.2 & 5.8 & 3.3 & -43.1 \\
\hline Delaware & 11.8 & 10.7 & -9.3 & 4.9 & 4.6 & -6.1 \\
\hline Florida* & 12.2 & 10.8 & -11.5 & 5.1 & 4.4 & -13.7 \\
\hline Georgia* & 12.8 & 12 & -6.2 & 6.5 & 4.9 & -24.6 \\
\hline Illinois & 14.2 & 12.5 & -12 & 5.1 & 5.4 & 5.9 \\
\hline Indiana & 16 & 14.3 & -10.6 & 6.6 & 6 & -9.1 \\
\hline lowa & 11.6 & 8.5 & -26.7 & 4.9 & 4.5 & -8.2 \\
\hline Kansas & 19 & 13.2 & -30.5 & 6.9 & 5.5 & -20.3 \\
\hline Kentucky & 12.7 & 12.6 & -0.8 & 6 & 6.4 & 6.7 \\
\hline Louisiana & 14.1 & 10.6 & -24.8 & 6.3 & 5.6 & -11.1 \\
\hline Maryland & 13.6 & 10.3 & -24.3 & 4.8 & 4.3 & -10.4 \\
\hline Massachusetts & 8.8 & 6.6 & -25 & 3.9 & 3.9 & 0 \\
\hline Michigan & 16.4 & 12.3 & -25 & 5.9 & 4.9 & -16.9 \\
\hline Minnesota* & 11.7 & 10.5 & -10.3 & 4.6 & 4 & -13 \\
\hline Mississippi & 13.9 & 10.9 & -21.6 & 6.8 & 5.9 & -13.2 \\
\hline Missouri & 16.5 & 11.2 & -32.1 & 5.9 & 5.1 & -13.6 \\
\hline Nevada* & 12.4 & 8.9 & -28.2 & 6.7 & 4.8 & -28.4 \\
\hline
\end{tabular}


Citation: Rati R (2017) Recent Changes in the Infant Mortality Rates of Blacks and Whites in the United States. Health Econ Outcome Res 3: 130.DOI: 10.4172/2471-268X.1000130

Page 4 of 4

\begin{tabular}{|c|c|c|c|c|c|c|}
\hline New Jersey & 11 & 7.5 & -31.8 & 3.9 & 3.5 & -10.3 \\
\hline New York* & 8.8 & 7.8 & -11.4 & 4.7 & 4.1 & -12.8 \\
\hline North Carolina & 15.1 & 12.1 & -19.9 & 6.3 & 5.1 & -19 \\
\hline Ohio* & 14.8 & 14 & -5.4 & 6.2 & 5.4 & -12.9 \\
\hline Oklahoma & 18 & 13.2 & -26.7 & 7.8 & 7 & -10.3 \\
\hline Pennsylvania* & 15.1 & 11.5 & -23.8 & 5.9 & 4.4 & -25.4 \\
\hline South Carolina & 13.7 & 10.1 & -26.3 & 6.1 & 4.6 & -24.6 \\
\hline Tennessee & 15.7 & 12.3 & -21.7 & 6.4 & 5.6 & -12.5 \\
\hline Texas* & 11.5 & 10.9 & -5.2 & 5.8 & 4.9 & -15.5 \\
\hline Virginia & 15.4 & 10.8 & -29.9 & 5.4 & 4.4 & -18.5 \\
\hline Washington* & 10.3 & 10.8 & 4.9 & 4.1 & 3.9 & -4.9 \\
\hline Wisconsin & 15.2 & 12.7 & -16.4 & 5.2 & 4.9 & -5.8 \\
\hline
\end{tabular}

Table 1: Comparison of infant mortality rates (IMR) for Blacks and Whites in the United States and the rates of decline, $2007-2014$.

An asterisk against a state indicates the rate of decline in White IMR is LARGER than in the Black IMR during the period.

The percentage change is calculated as [(IMR2014/IMR2007)-1] $\times$ 100.

\section{References}

1. https://cia.gov/library/publications/the-world-factbook/

2. Annie E (2017) Casey foundation. Kids Count Data Center.

3. SACIM Subcommittee on Eliminating Health Disparities (2006) Eliminating health disparities in infant mortality.
4. National Center for Health Statistics (2012) Healthy People 2010 Final Review, Hyattsville, MD, USA.

5. Ram R (2011) The inverse care law and infant mortality among Whites and Blacks in the United States. Inter J Soc Econ 38: 973-982.

6. US Census Bureau (2012) Statistical Abstract of the United States: 2012. Washington, DC, USA.

7. Mayer S, Sarin A (2005) Some mechanisms linking economic inequality and infant mortality. Soc Sci Med 60: 439-455.

8. Cutler DM, Meara E, Seth RS (2012) Induced innovation and social inequality: evidence from infant medical care. J Hum Resour 47: 456-492.

9. Child Trends Database (2016) Infant, child and teen mortality. 